\title{
The Small GTP-Binding Protein TC10 Promotes Nerve Elongation in Neuronal Cells, and Its Expression Is induced during Nerve Regeneration in Rats
}

\author{
Katsuhisa Tanabe, ${ }^{1,2,3,6}$ Taro Tachibana, ${ }^{4}$ Toshihide Yamashita, ${ }^{3}$ Yong Ho Che, ${ }^{2,3,6}$ Yoshihiro Yoneda, ${ }^{4}$ \\ Takahiro Ochi, ${ }^{5}$ Masaya Tohyama, ${ }^{3,6}$ Hideki Yoshikawa, ${ }^{2}$ and Hiroshi Kiyama ${ }^{1,6}$ \\ ${ }^{1}$ Department of Anatomy, Asahikawa Medical College, Asahikawa, 078-8510 Japan, Departments of 2Orthopaedic \\ Surgery, ${ }^{3}$ Anatomy and Neuroscience, ${ }^{4}$ Anatomy and Cell Biology, and 5 Applied Medical Engineering, Graduate School of \\ Medicine, Osaka University, Osaka, 565-0871 Japan, and ${ }^{6}$ Core Research for Evolutional Science and Technology \\ (CREST), Japan Science and Technology (JST), Kawaguchi, Saitama, 332-0012 Japan
}

\begin{abstract}
We have made a rat cDNA library using nerve-transected hypoglossal nuclei. Using this library, we performed expressedsequence tag analysis coupled with in situ hybridization to identify genes whose expression is altered in response to nerve injury. In this gene screening, a member of Rho family GTPases, TC10, which had not yet been characterized in neuronal cells, was identified. TC10 mRNA expression was very low in normal motor neurons; however, axotomy induced its expression dramatically. Other family members such as RhoA, Rac1, and Cdc42 were moderately expressed in normal motor neurons and showed slight upregulation after axotomy. The expression level of TC10 mRNA was low in the embryonic brain and
\end{abstract}

gradually increased with development. However, the expression of TC10 mRNA in the adult brain was lower and more restricted than that of RhoA, Rac1, and Cdc42. Cultured dorsal root ganglia exhibited dramatic neurite extension secondary to adenovirus-mediated expression of TC10. It can be concluded that although TC10 expression is lower in developing and mature motor neurons compared with other Rho family members, TC10 expression is induced by nerve injury to play a crucial role in nerve regeneration, particularly neurite elongation, in cooperation with other family members.

Key words: Rho; nerve regeneration; cytoskeleton; axotomy; adenovirus; hypoglossal motor neuron; DRG
Neurons in the peripheral nervous system (PNS) are able to survive and regenerate after nerve injury, whereas most of the neurons in the CNS die after nerve injury. This is one of the major differences between CNS and PNS neurons. Identification of the molecular basis of this difference is expected to lead to opportunities to rescue injured CNS neurons. In injured neurons, expression of various kinds of molecules occurs in response to nerve injury. The precisely regulated expression of molecules might be vital for proper nerve regeneration. To explore the molecular mechanism underlying neuronal regeneration, we have attempted to identify molecules whose mRNA expression is altered in response to nerve injury (Kitahara et al., 1994; Kiryu et al., 1995a,b; Morita et al., 1996; Yao et al., 1997; Namikawa et al., 1998; Tanabe et al., 1998). In these efforts, we have recently performed expressed-sequence tag (EST) analysis by making a special cDNA library derived from the hypoglossal nuclei after axotomy, which is an effective method to identify target molecules (Tanabe et al., 1999). In that study, several known and unknown genes whose expression is upregulated after axotomy were identified. Among those clones, a Rho family GTPase, TC10, was retrieved. TC10 was originally cloned from a human teratocarci-

\footnotetext{
Received Jan. 27, 2000; revised March 13, 2000; accepted March 23, 2000.

This work was supported in part by a Grant-in-Aid for Scientific Research from the Ministry of Education, Science and Culture, Japan, and the Ministry of Health and Welfare, Japan. K.T. is a fellow of Japan Society for the Promotion of Science. We are indebted to Dr. Y. Takai for supplying plasmids of RhoA, Rac1, and Cdc42, and to I. Saito and K. Namikawa for adenovirus vectors.

Correspondence should be addressed to Prof. Hiroshi Kiyama, Department of Anatomy, Asahikawa Medical College, 2-1-1-1 Midorigaoka-Higashi, Asahikawa, Hokkaido, 078-8510, Japan. E-mail: kiyama@asahikawa-med.ac.jp.

Copyright (C) 2000 Society for Neuroscience $0270-6474 / 00 / 204138-07 \$ 15.00 / 0$
}

noma cDNA library (Drivas et al., 1990) and is most similar to Cdc42 and Rac1. This molecule has not been characterized as well as other Rho family GTPases such as RhoA, Rac1, and Cdc42 (Neudauer et al., 1998); in particular, no evidence is available regarding its function in neuronal cells. Rho family GTPases are known in particular as regulators of cell morphology by cytoskeletal organization. In fibroblasts, $\mathrm{Cdc} 42$ induces filopodia, Rac1 induces lamellipodia, and RhoA induces actin stress fibers, respectively (Ridley and Hall, 1992; Ridley et al., 1992; Nobes and Hall, 1995). In N1E-115 neuroblastoma cells, Cdc42 and Rac1 induce lamellipodia, cell spreading, and neurite-like spike formation (Leeuwen et al., 1997). In contrast, RhoA induces cell rounding and cortical shell actin formation (Kranenburg et al., 1997). In the growth cone of N1E-115 cells, Cdc42 induces filopodia followed by lamellipodia, Rac1 induces lamellipodia, and RhoA induces collapse of the growth cone (Kozma et al., 1997). In embryonic chick neurons, Cdc42, Rac1, and RhoA regulate growth cone collapse and neurite growth inhibition induced by CNS myelin and collapsin-1 (Jin and Strittmatter, 1997; Kuhn et al., 1999). These findings suggest strongly that Rho family members are implicated in growth cone behavior and neurite elongation. In this respect, it is conceivable that TC10 plays some pivotal role in nerve regeneration. As for TC10 function, it has been reported that TC10 triggers microspike formation in a fashion similar to $\mathrm{Cdc} 42$ in NIH $3 \mathrm{~T} 3$ fibroblasts (Neudauer et al., 1998). However, there are no reports clarifying how TC10 regulates growth cone locomotion or neurite outgrowth. In peripheral nerve regeneration, the distal end of an axon forms a growth cone and its locomotion is essential for axonal elongation to its target. It is therefore assumed that Rho 
family GTPases might be implicated in the regeneration process, regulating growth cone formation and/or locomotion. In this study, we compared the expression change of major representatives of the Rho family GTPases, RhoA, Rac1, and Cdc42, with that of TC10 after peripheral nerve axotomy and found that the change of expression of TC10 was the most marked among the family proteins. Characterization of TC10 was also attempted, in particular regarding neurite elongation in organ-cultured dorsal root ganglia (DRG), using an adenovirus-mediated gene expression system.

\section{MATERIALS AND METHODS}

Cloning of rat TC10 cDNA with cDNA library screening. A rat brain cDNA library (Lambda ZAPII library, Stratagene, La Jolla, CA) was screened with a subcloned $739 \mathrm{bp}$ DNA fragment by means of plaque hybridization. The probe was labeled with $\alpha{ }^{32} \mathrm{P} \mathrm{dCTP}$ using random oligoprimers and klenow fragment. Approximately $1 \times 10^{6}$ plaques were screened and hybridized in $40 \%$ formamide, $4 \times$ SSC, 0.04 m sodium phosphate, $8 \times$ Denhardt's solution, and $0.8 \%$ SDS at $42^{\circ} \mathrm{C}$, and washing was performedin $1 \times \mathrm{SSC}$ and $0.8 \%$ SDS at $50^{\circ} \mathrm{C}$. Positive plaques were obtained and purified using in vivo excision for plasmids. Both strands of these cDNA inserts were sequenced with a dye primer and dye terminator cycle sequencing kit (Applied Biosystems, Foster City, CA) using an Applied Biosystems model 377 DNA sequencer.

In situ hybridization. Operations on animals and in situ hybridization were performed as described previously (Tanabe et al., 1998, 1999). The right hypoglossal nerve of male Wistar rats weighing $100 \mathrm{gm}$ was transected, and brain sections were made $1-56 \mathrm{~d}$ after the operation. To detect the distribution of Rho family proteins, male Wistar rats weighing $150 \mathrm{gm}$ were used. RhoA, Rac1, and Cdc42 cDNA were donated by Dr. Y. Takai (Osaka University, Osaka, Japan). Rho, Rac, Cdc42, and TC10 cDNA were cloned into pBluescript SK vectors. They were linearized by cutting at a single site, and in vitro transcription was performed using T7 and T3 RNA polymerase and $\left[\alpha^{-}{ }^{35}\right.$ S $]$ UTP (Dupont-NEN, Wilmington, DE) to prepare both antisense and sense riboprobes. The findings were assessed using both film autoradiography and emulsion autoradiography. The grain intensity on the $\mathrm{x}$-ray film was measured using a computerized image analysis system (MCID: Image Res., Ontario, Canada). For statistical analysis, eight sections each from three rats were studied.

Immunohistochemistry with polyclonal anti-TC10 antibody. TC10 polyclonal antibody was raised against rat TC10 partial peptide (184-198: TPKKHTVKKRIGSRC). Antibodies were purified from immune rabbit serum by affinity chromatography using ProtOn kit 1 (Multiple Peptide Systems). Immunoglobulins were concentrated by ammonium sulfate cut from preimmune serum. Western blotting revealed that TC10 recombinant protein and endogenous TC10 protein in PC12 cells can be detected by this anti-TC10 antibody but not by preimmune immunoglobulins. Fixed brain stem sections were blocked with PBS containing $1 \%$ bovine serum albumin and $2 \%$ normal goat serum (PBS/BSA/NGS) for $1 \mathrm{hr}$ at room temperature. Then tissues were incubated with immunoglobulins from preimmune serum or from immune serum for $16 \mathrm{hr}$ at $4^{\circ} \mathrm{C}$ and washed three times with PBS and incubated with Cy3-labeled goat anti-rabbit antibodies (dilution 1:500) (Amersham Pharmacia Biotech) for $1 \mathrm{hr}$ at room temperature. Finally, they were washed three times and viewed and photographed with a Zeiss Axiophot 2 microscope.

Western blot analysis. PC12 whole-cell lysates were resolved by SDSPAGE and electroblotted onto Immobilon $\mathrm{P}$ membranes (Millipore, Bedford, MA). After blocking, the membranes were probed with antiTC10 antibody or preimmune immunoglobulin. Immunocomplexes were visualized using enhanced chemiluminescence detection (Amersham Pharmacia Biotech).

Northern blot analysis. Total RNA of various adult rat tissues was isolated using RNAeasy kit (Qiagen, Hilden, Germany). First, $20 \mu \mathrm{g}$ RNA from each tissue was separated on $1 \%$ agarose formamide gels and transferred onto nylon membranes (Hybond-N, Amersham Pharmacia Biotech). As a template, the TC10 cDNA fragment (bases 307-946) was labeled and hybridized following the same procedure for plaque hybridization described above. After hybridization with the TC10 probe, the same membrane was rehybridized with a glyceraldehyde-3-phosphate dehydrogenase probe.

Construction of adenovirus vectors. Mutagenesis of Gly to Val at codon 18 in activated (V18) TC10 was performed by site-directed mutagenesis using a mutagenesis kit (Stratagene). The V18TC10 cDNA was tagged at

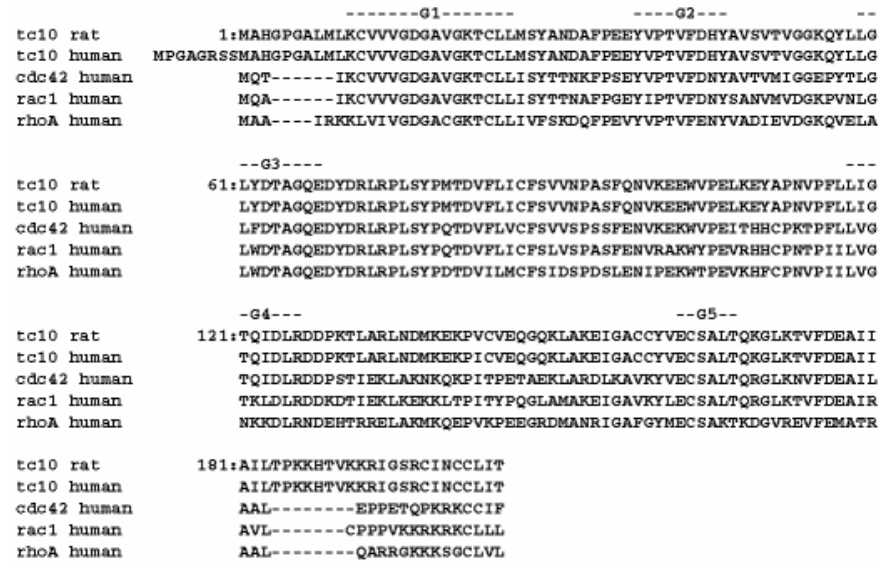

Figure 1. Alignment of rat TC10 compared with human TC10, Cdc42, Rac1, and RhoA. G1-G5 indicate Ras canonical boxes.

the 5' end using the PCR with a DNA sequence encoding the flag epitope DYKDDDDK and subcloned into pAdex1CAwt cosmid vectors. The expression cosmid cassette and the adenoviral DNA-terminal protein complex were cotransfected into 293 cells via calcium phosphate precipitation. Incorporation of the expression cassette into the isolated recombinant virus was confirmed by digestion with appropriate restriction enzymes. The recombinant virus, AdexCAFlagV18TC10, was subsequently propagated in 293 cells, and the viral suspension was stored at $-80^{\circ} \mathrm{C}$. For control experiments, we used a nuclear-localizing $\beta$-galactosidase-expressing adenovirus, AdexCANLacZ, which was provided by I. Saito (Tokyo University, Tokyo, Japan). Multiplicity of infection, which represents the number of plaque-forming units per cell, was calculated based on titration of 293 cells (Namikawa et al., 2000).

Rat DRG organ culture and adenoviral infection. Lumbar DRG were removed aseptically from postnatal day 0 (P0) rats and pooled in ice-cold L15 medium. The DRG were incubated with $15 \mu \mathrm{l}$ of DMEM with 5\% FCS containing AdexCAFlagV18TC10 or AdexCANLacZ at a concentration of $5 \times 10^{8} \mathrm{pfu} / \mathrm{ml}$ for $1 \mathrm{hr}$ at $37^{\circ} \mathrm{C}$. After incubation with adenovirus vector, the adenovirus-containing medium was aspirated, and DRG were buried in $0.4 \%$ collagen type 1 gel (KOKEN, Tokyo, Japan). After the gel had consolidated, DMEM/F12 mixture (1:1) with N2 supplement (Life Technologies, Gaithersburg, MD), $5 \mu \mathrm{M}$ uridine, and 5 $\mu \mathrm{M}$ fluorodeoxyuridine was added, and the ganglia were cultured in $5 \%$ $\mathrm{CO}_{2}$ at $37^{\circ} \mathrm{C}$.

Immunofluorescence of cultured DRG. AdexCAFlagV18TC10-infected ganglia were fixed with $4 \%$ paraformaldehyde in PBS for $20 \mathrm{~min}$, washed, and permeabilized with $0.2 \%$ Triton X-100 in PBS for 5 min. After a brief washing step, fixed ganglia were blocked with PBS containing PBS/BSA/NGS for $1 \mathrm{hr}$ at room temperature. Primary antibody incubations were performed in PBS/BSA/NGS for $1 \mathrm{hr}$ at room temperature or for $14-16 \mathrm{hr}$ at $4^{\circ} \mathrm{C}$. Flag-tagged TC10 was detected using anti-Flag M2 monoclonal antibody (dilution 1:500) (Eastman Chemical). Antineurofilament mouse antibody was from $2 \mathrm{H} 3$ hybridoma (Developmental Studies Hybridoma Bank, The Johns Hopkins University, Baltimore, $\mathrm{MD})$. The cells with primary antibodies were washed three times with PBS and then incubated with Alexa488-labeled goat anti-mouse antibodies (dilution 1:250) (Molecular Probes, Eugene, OR) for $1 \mathrm{hr}$ at room temperature for detection of primary antibodies. AdexCANlacZ-infected DRG were fixed with $2 \%$ paraformaldehyde and $0.05 \%$ glutaraldehyde in PBS for $5 \mathrm{~min}$ at $4^{\circ} \mathrm{C}$. Infected cells were identified using the standard $\beta$-galactosidase conversion assay. Finally, DRG were viewed on a Zeiss LSM510 laser confocal microscope, and the lengths of neurites were measured.

Measurement of neurite length. Twenty DRG infected with AdexCAFlagV18TC10 and 20 infected with AdexCANlacZ were compared. The length of the longest 20 neurites of each ganglion was measured from the periphery of the ganglia using Zeiss LSM510 software, and the average length of 400 neurites in each group was calculated.

Database accession number. The nucleotide sequence of rat TC10 cDNA will appear in DNA Database of Japan (DDBJ), European Mo- 


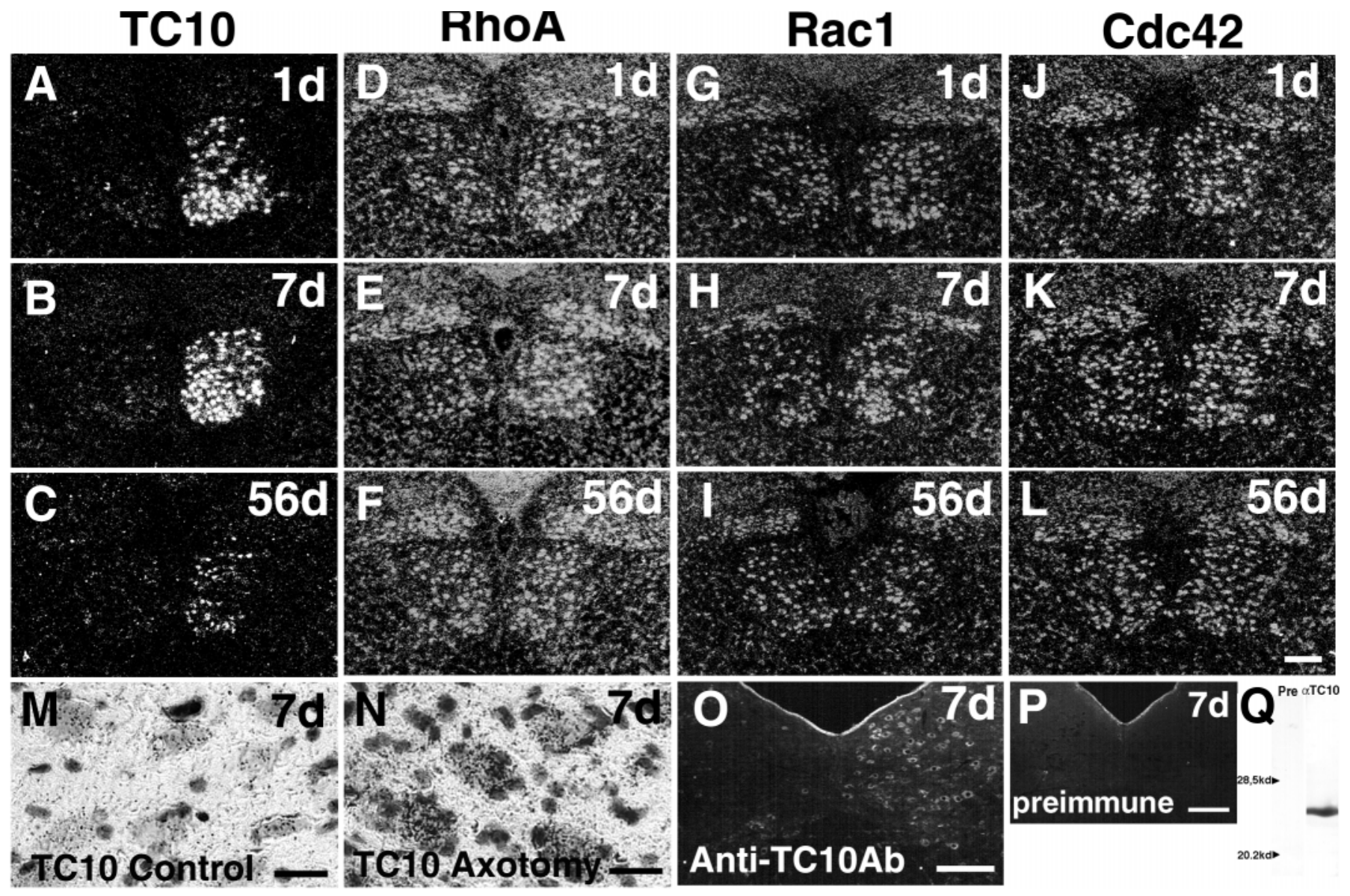

Figure 2. Expression of mRNA of TC10 $(A-C)$, RhoA $(D-F)$, Rac1 $(G-I)$, and $\operatorname{Cdc} 42(J-L)$ in hypoglossal nucleus 1,7 , and $56 \mathrm{~d}$ after unilateral hypoglossal nerve transection (right side). All four Rho family GTPases showed increased expression after axotomy. The change in expression of TC10 was most significant. Bright-field micrograph stained with thyonin shows the localization of TC10 mRNA in normal $(M)$ and axotomized $(N)$ hypoglossal nuclei $7 \mathrm{~d}$ after operation. Note the high density of grains in the large neurons on the axotomized side. Immunoreactivity of TC10 in hypoglossal nuclei $7 \mathrm{~d}$ after axotomy is shown in $O$. Protein expression of TC10 as well as its mRNA increased in the hypoglossal neurons after axotomy. Control preimmune staining pattern is shown in $P$. Western blot analysis of PC12 cell lysate with anti-TC10 antibody showed a single band, which was not detected with preimmune immunoglobulin $(Q)$. Scale bars: $A-L, O, P, 250 \mu \mathrm{m} ; M, N, 50 \mu \mathrm{m}$.

lecular Biology Laboratory, and GenBank nucleotide sequence databases with the accession number AB031482.

\section{RESULTS}

Cloning of rat TC10 with EST approach using the axotomized hypoglossal nuclei-derived cDNA library

Previously, we have established a special cDNA library, which was made using rat injured hypoglossal nuclei (Tanabe et al., 1999). In this study, we identified two distinct but partially overlapping unknown clones among the $750 \mathrm{cDNA}$ clones, which were subcloned randomly from the cDNA library. An in situ hybridization study revealed strong upregulation of the mRNA expression in axotomized hypoglossal motor neurons. Subsequently we screened a rat brain cDNA library to isolate the full coding region of this clone. Among the $1 \times 10^{6}$ clones of the rat brain cDNA library that were screened, 46 positive plaques were identified. A search through the DDBJ showed that this clone has high homology with the human TC10 gene. The human TC10 protein encodes 205 or 213 amino acids, depending on the choice of the first methionine. This rat homolog contains an open reading frame of 205 amino acids that predicts a $23 \mathrm{kDa}$ protein and shows an almost complete match with the shorter human TC10, except for one amino acid exchange (Val to Ile at 143) (Fig. 1). In addition, all functional domains of Rho family proteins were preserved in this rat homolog. Human TC10 has $67.4 \%$ identity with $\mathrm{Cdc} 42$, $64.7 \%$ with Rac1, and $52.2 \%$ with RhoA.

\section{Expression profile of rat TC10 during nerve regeneration}

We performed in situ hybridization to evaluate changes in the expression of rat TC10 over time during nerve regeneration (Figs. $2 A-C, 3 A)$. The expression level of TC10 mRNA was very low in the uninjured hypoglossal nucleus; however, it became very high on the injured side $1 \mathrm{~d}$ after axotomy, and the signal intensity increased by postoperative day 7 . Thereafter it decreased gradually, but a more intense signal was detected on the operated side than the control side even $56 \mathrm{~d}$ after axotomy. Expression of the TC10 mRNA signal was limited to the injured motor neurons and was not observed in the surrounding glial cells at any time after axotomy (Fig. $2 M, N$ ). Immunoreactivity of TC10 was examined using a polyclonal antibody raised against a TC10 partial peptide. Protein expression of TC10 was also induced in the hypoglossal motor neurons in response to axotomy (Fig. 2O). No expression was detected in the brain stem section after axotomy using preimmune immunoglobulins (Fig. 2P). Western blot analysis with PC12 cell lysate revealed that TC10 could be detected as a single 

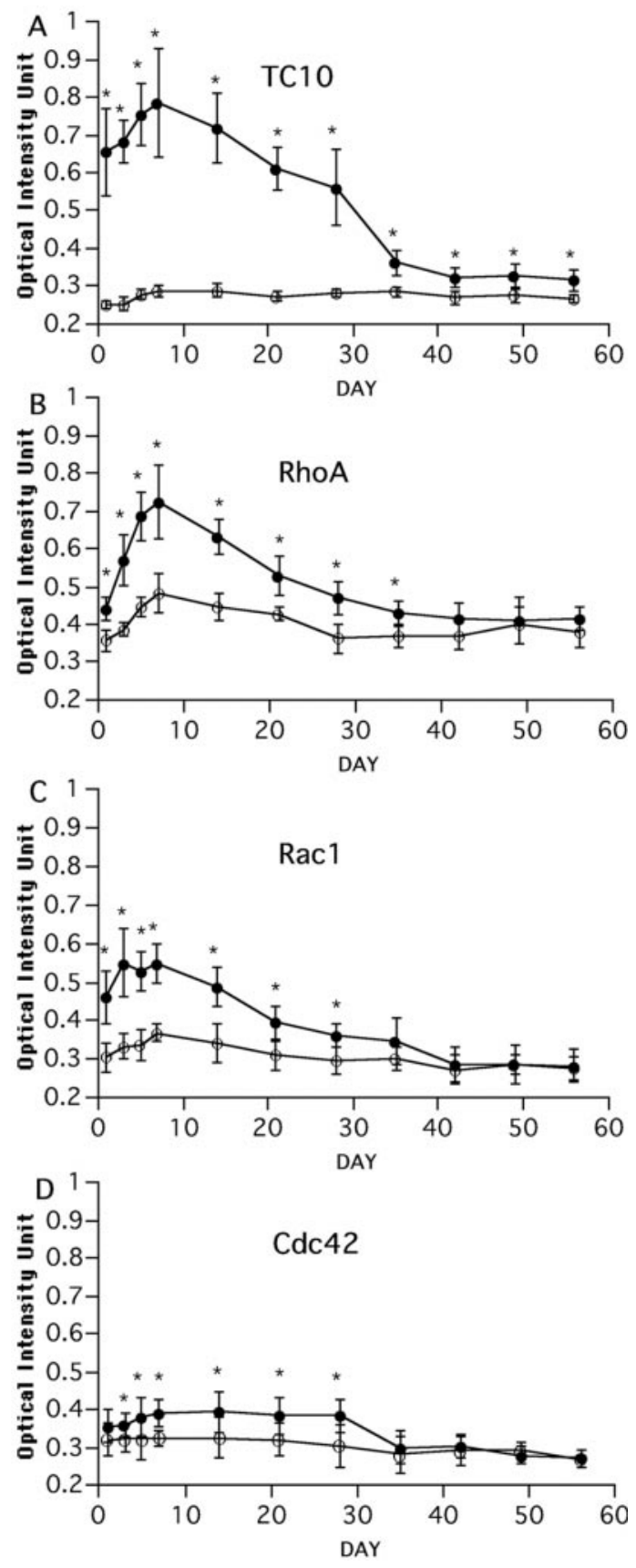

Figure 3. Expression profile of mRNA of TC10 $(A)$, RhoA $(B)$, Rac1 $(C)$, and $\operatorname{Cdc} 42(D)$ on transection side $(\bullet)$ and control side $(O)$. Eight sections each from three rats were studied for statistical analysis. Each point shows the mean and SD intensity of the positive signals. Asterisks denote statistically significant differences (Student's $t$ test) from control: ${ }^{*} p<0.05$.

band with this anti-TC10 antibody, and not with preimmune immunoglobulins (Fig. 2Q).

Although TC10 is one of the Rho GTPase family members of the Ras superfamily, recent studies of Rho family proteins were mainly focused on $\mathrm{Cdc} 42$, Rac1, and RhoA. These three proteins were demonstrated to be essential for the regulation of growth cone motility. We therefore examined whether mRNA of these family proteins is expressed in hypoglossal motor neurons and whether their expression is also altered after peripheral nerve axotomy. The in situ hybridization study revealed that the expres- sion level of these proteins was higher than that of TC10 in intact motor neurons, and that there was only a slight, if any, increase in $\mathrm{Cdc} 42$, Rac1, and RhoA expression after injury (Figs. $2 D-L$, $3 B-D)$. The upregulation of $\mathrm{Cdc} 42$ mRNA expression was just above the significant level. The change in mRNA expression of $\mathrm{TC} 10$ in response to axotomy was much more marked than that for $\mathrm{Cdc} 42$.

\section{TC10 mRNA localization in brain and various other tissues}

Localization of mRNA for TC10, RhoA, Rac1, and Cdc42 in the adult rat brain was studied by means of in situ hybridization (Fig. 4). As described previously, RhoA, Rac1, and Cdc42 were ubiquitously expressed in the adult brain, including the hippocampus, neocortex, thalamus, and cerebellum (Threadgill et al., 1997). In contrast, weak TC10 mRNA expression was found in the CA1 region of the hippocampus, the superior and inferior colliculi, and some brain stem nuclei (Fig. $4 A$ ). Hybridization with the sense RNA probes revealed no signal at all (data not shown). Northern blot analysis revealed that TC10 is ubiquitously expressed in various tissues (Fig. 5A). TC10 expression was very high in skeletal muscle, moderate in brain, heart, lung, and placenta, and low in liver, kidney, colon, ovary, and testis. TC10 expression in the brain increased with development from the fetal to the adult brain. The demonstration of TC10 mRNA expression in the E16 whole fetus by in situ hybridization confirmed that expression in the fetal brain and spinal cord was very low (Fig. 5B), whereas TC10 was ubiquitously expressed in various tissues except the CNS.

\section{TC10 promotes neurite extension in rat DRG cells}

We evaluated whether V18TC10 promotes neurite elongation using DRG organ culture. Before examining this, we confirmed that upregulation of TC10 mRNA expression occurs in sensory ganglia. L5 DRG of the adult rat was examined after sciatic nerve transection. Expression of TC10 on the nonoperated side was very low (Fig. $6 A$ ), whereas sciatic nerve transection significantly induced the expression of TC10 mRNA in DRG neurons as well as hypoglossal neurons (Fig. 6B). Next, a gain-of-function mutant V18TC10 was constructed, and Flag tag was attached at its N-terminal end (Flag-V18TC10). We further inserted FlagV18TC10 into an adenovirus vector (AdexCAFlagV18TC10). DRG was isolated from neonatal rats (P0), infected with AdexCAFlagV18TC10 or AdexCANLacZ, buried in collagen gel, and cultured in serum-free medium. Seven days after infection, the lengths of neurites were compared between both groups. AdexCAFlagV18TC10-infected DRG had apparently longer neurites than AdexCANLacZ-infected DRG (Fig. 6C-E). The expression of transfected proteins was confirmed using Flag immunostaining and $\beta$-galactosidase assay (data not shown).

\section{DISCUSSION}

In the present study, TC10 was identified as a nerve injuryassociated molecule in our gene screening; however, TC10 had not been characterized in neuronal cells. Therefore we attempted to characterize TC10 in cultured DRG and nerve-injured rats. The present findings suggest a strong relationship between TC10 expression and nerve regeneration.

\section{Isolation of TC10 as a nerve injury-associated protein}

In a previous EST study using a nerve-injured motor nucleiderived cDNA library, we selected approximately 750 clones randomly and sequenced them (Tanabe et al., 1999). Two clones 

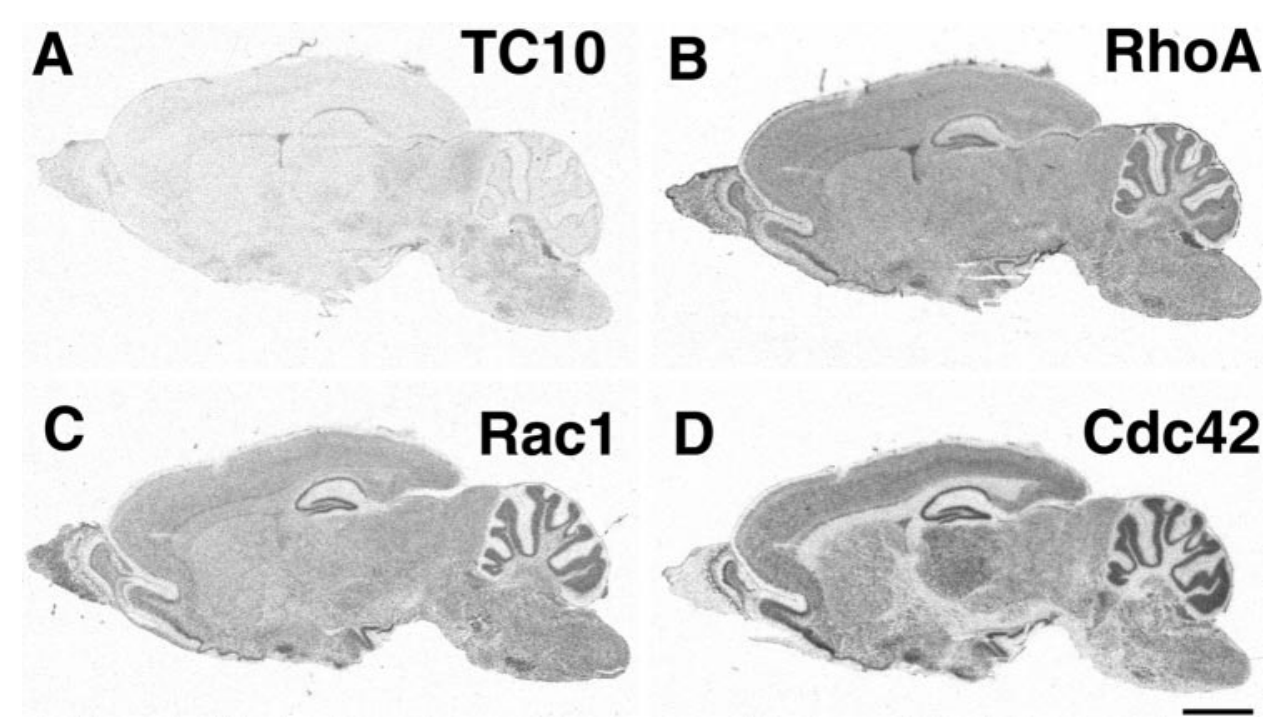

Figure 4. Expression of mRNA of TC10 $(A)$, RhoA $(B)$, Rac1 $(C)$, and Cdc42(D) in rat brain. Expression of TC10 is limited to some areas, including some nuclei of the brain stem, superior and inferior colliculus, and CA1 region of the hippocampus, but was not detected in the cerebral cortex. However, other Rho family GTPases are ubiquitously expressed in the brain. Scale bar, $3 \mathrm{~mm}$.
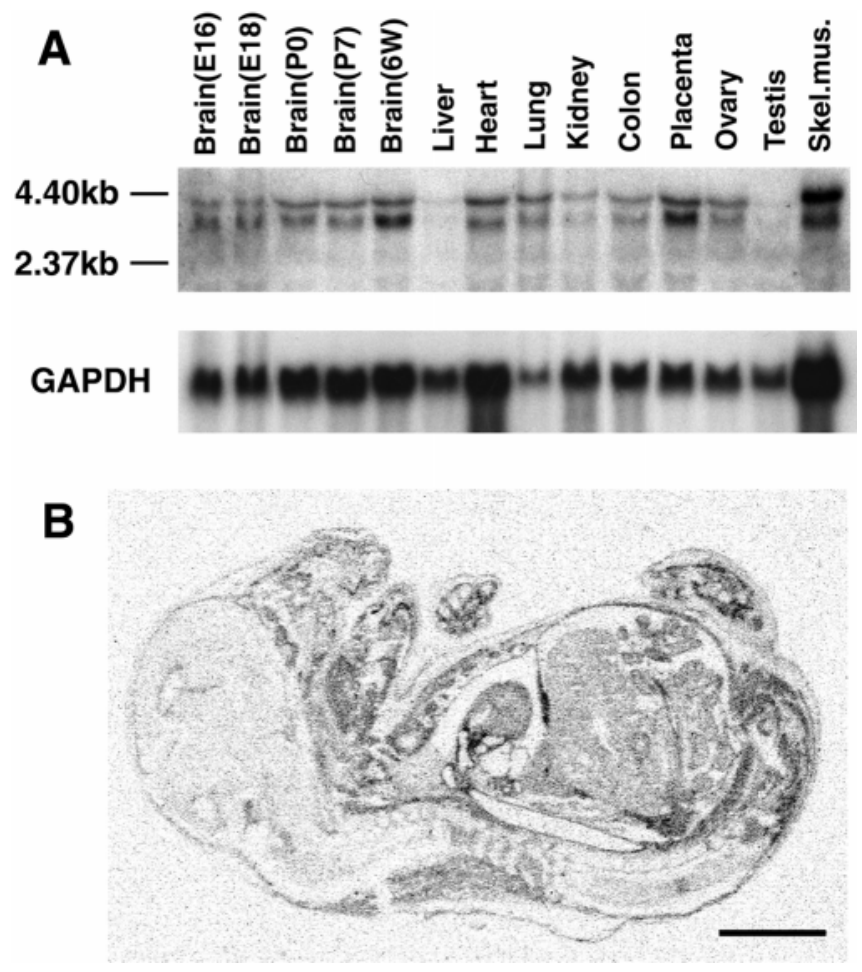

Figure 5. A, mRNA expression of TC10 in embryonic day 16 (E16), E18, $\mathrm{P} 0$, and $\mathrm{P} 7$ rat brains and various tissues of the adult $(P 6 W)$ rat. TC10 expression in developing brain is lower than that in adult brain. High expression is observed in skeletal muscle among various organs. mRNA level was confirmed by hybridization with glyceraldehyde-3-phosphate dehydrogenase $(G A P D H)$. B, mRNA expression of TC10 in rat E16 fetus. Expression in brain and spinal cord was low in contrast to the ubiquitous expression in other tissues. Scale bar, $3 \mathrm{~mm}$.

coding TC10 cDNA were identified; however, other members of the Rho family GTPases were not obtained. This suggests that TC10 may be more abundant in the nerve-injured motor nucleiderived cDNA library. The present in situ hybridization study also supports the significance of TC10 expression in nerve-injured hypoglossal motor neurons. In normal hypoglossal motor neurons, other Rho family molecules were expressed to some extent, whereas the expression level of TC10 was almost below the detection limit. However, when a nerve is injured, the response in TC10 expression is dramatic. The mRNA expression of other Rho family members such as RhoA, Rac1, and Cdc42 is also upregulated after axotomy, although the magnitude of the responses is less than that of TC10. This intriguing contrast between TC10 and the other members in injured motor neurons suggests an important role of TC10 in nerve regeneration. In addition, the induced expression of TC10 together with other Rho family GTPases after axotomy might be important for neurite outgrowth and growth cone morphology. In peripheral nerve regeneration, axons extend along the original basal lamina of Schwann cells, which are rich in cell adhesion molecules such as laminin, N-cadherin, L1, and N-CAM (Martini and Schachner, 1988; Martini, 1994). It is proposed that the growth cones adhere to the basal lamina via these adhesion molecules and advance with extensions of filopodia and lamellipodia (Bentley and O’Connor, 1994; Lin et al., 1994). Rho family GTPases regulate the advance and collapse of growth cones to lead them into the ideal Schwann tubes and then to the target organ. It was reported that wild-type TC10 produces no phenotype in fibroblasts (Neudauer et al., 1998). The induction of TC10 expression by axotomy indicates that neurons may contain a TC10-specific exchange factor that is lacking in fibroblasts. The present study suggests that the cooperative function of Rho family members, not only TC10 but also RhoA, Rac1, and Cdc42, might be crucial to coordinate growth cone behavior such as motility, adhesion, and sensory capacity.

\section{TC10 is not abundant in the developing brain compared with other Rho family members}

Northern blot analysis revealed that TC10 is ubiquitously expressed in various rat tissues. Two bands were displayed in all the TC10-expressing tissues. The length of TC10 cDNA that we identified was $3858 \mathrm{bp}$ (excluding the polyA tail), which was longer than the shorter band. Northern blot analysis of TC10 in human tissues also showed two bands (Neudauer et al., 1998). 

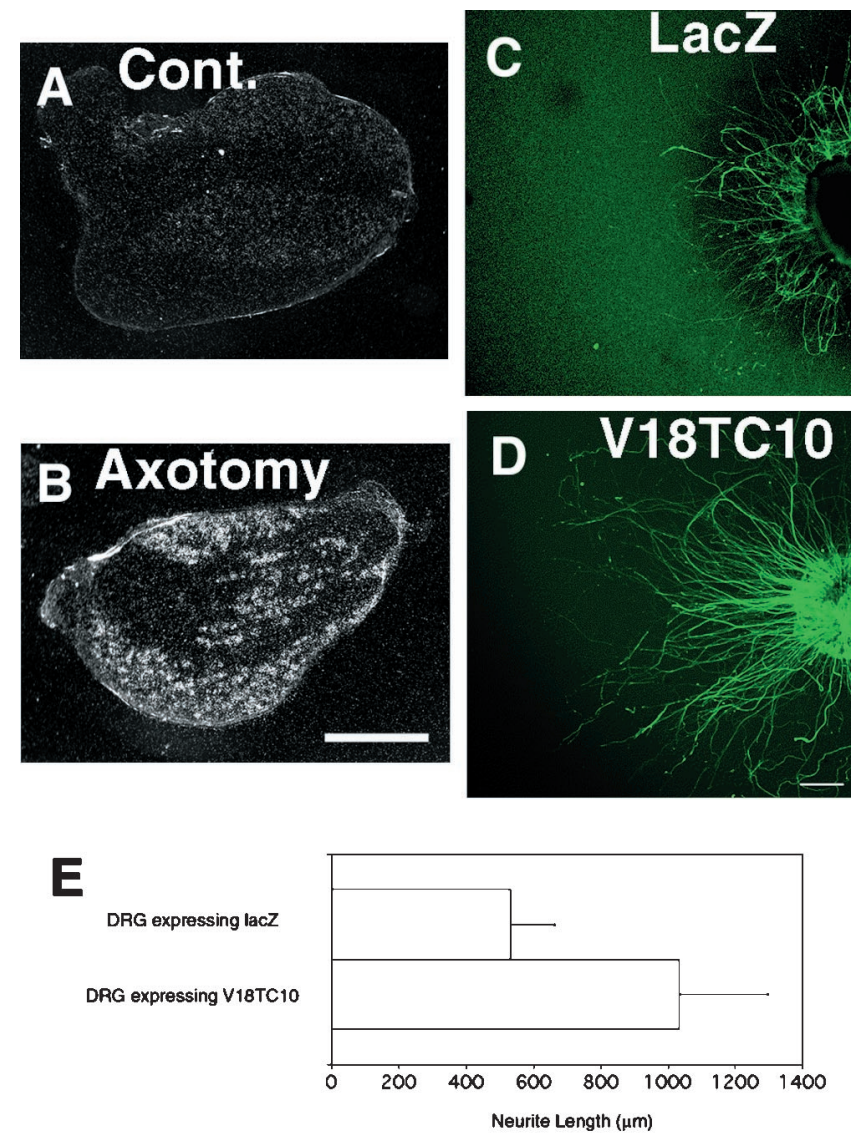

Figure 6. $A, B$, mRNA expression of TC10 in left $(A)$ and right $(B) \mathrm{L} 5$ DRG $4 \mathrm{~d}$ after right sciatic nerve transection. mRNA expression was induced in DRG neurons in response to axotomy. Scale bar, $500 \mu \mathrm{m} . C$, $\mathrm{D}, \mathrm{DRG}$ were infected with AdexCANlacZ $(C)$ or AdexCAV18TC10 $(D)$ and then buried in collagen gel. Seven days after infection, they were fixed, and neurites were visualized with anti-neurofilament antibody. Overexpression of V18TC10 increased the length of neurites of DRG cells. Scale bar, $50 \mu \mathrm{m}$. $E$, Neurite length was measured from the surface of DRG. Error bars represent SD.

Therefore, alternative transcription may contribute to the two bands. Ubiquitous expression of TC10 may depend on diverse functions of TC10. Several effector molecules of TC10 have been detected (Neudauer et al., 1998), and TC10 stimulates Jun $\mathrm{N}$-terminal kinase and PAK kinase activation. Although little is known about the expression pattern or pivotal roles of these effectors in vivo, their numerousness indicates diverse functions of TC10. In human tissues, TC10 expression is very high in skeletal muscle and heart and is low in other organs (Neudauer et al., 1998). Our study also demonstrated high expression in skeletal muscle and heart. In cardiomyocytes, RhoA function is required for $\alpha 1$-adrenergic receptor-stimulated atrial natriuretic factor and myosin light chain-2 expression and myofibrillar organization (Sah et al., 1996; Hoshijima et al., 1998). In skeletal muscle, Rho family GTPases regulate transcription of muscle-specific genes, which are involved in muscle differentiation (Carnac et al., 1998; Takano et al., 1998). In muscle tissue, TC10 may play an important role as well as other Rho family GTPases. In the adult brain, the expression of TC10 was slightly higher than in the developmental stages. The moderate expression in the adult brain suggests that TC10 may regulate neuronal plasticity by modulating growth cone formation. In the embryo, TC10 expression was very low in the brain, whereas ubiquitous expression was observed in other tissues. RhoA, Rac1, and Cdc42 are abundantly expressed in the embryonic brain, and their central role in morphological development of cortical cells is reported (Threadgill et al., 1997). Although functional similarity between $\mathrm{Cdc} 42$ and TC10 has been described, the developmental expression profile is different. Therefore, Cdc42 may play a pivotal role in development, whereas TC10 may play another pivotal role in nerve regeneration. The contrasting responses to nerve injury of TC10 and Cdc42 also suggest that TC10 may be involved in regenerative neurite elongation but not in developmental neurite elongation.

\section{TC10 promotes neurite extension of rat DRG}

The role of Rho family members in neurite extension and growth cone morphology of PNS neurons is complicated. In embryonic chick neurons, Rac1 and RhoA repress growth cone collapse and neurite growth inhibition induced by CNS myelin (Kuhn et al., 1999). However, Cdc42 and Rac1 mediate collapsin-1-induced growth cone collapse and neurite growth inhibition (Jin and Strittmatter, 1997; Kuhn et al., 1999). The inhibition of RhoA with C3 transferase stimulates neurite outgrowth (Jin and Strittmatter, 1997). To clarify the function of TC10 in the nervous system, DRG organ culture was used. Before construction of this experimental paradigm, regulation of TC10 mRNA expression in the DRG after sciatic nerve transection was examined. TC10 mRNA expression was also substantially induced in DRG neurons in response to axotomy. Subsequently, we evaluated the effect of the gain-of-function TC10 expression in DRG. In the organ culture, TC10 promoted significant neurite extension of DRG neurons. Although the precise mechanism underlying how TC10 promotes neurite elongation is unclear, organ culture appears to reflect in vivo peripheral nerve regeneration better than previous cell culture studies.

\section{Conclusions}

The present study characterized TC10, a small GTP-binding protein in neuronal cells. In cultured DRG, TC10 promotes neurite elongation. TC10 mRNA expression is strongly induced in nerve-injured hypoglossal motor neurons and DRG sensory neurons during the nerve regeneration process, whereas TC10 expression in the embryonic brain is lower. These findings suggest that TC10 promotes axonal outgrowth, especially in nerve regeneration.

\section{REFERENCES}

Bentley D, O'Connor TP (1994) Cytoskeletal events in growth cone steering. Curr Opin Neurobiol 4:43-48.

Carnac G, Primig M, Kitzmann M, Chafey P, Tuil D, Lamb N, Fernandez A (1998) RhoA GTPase and serum response factor control selectively the expression of MyoD without affecting Myf5 in mouse myoblasts. Mol Biol Cell 9:1891-1902.

Drivas GT, Shih A, Coutavas E, Rush MG, D'Eustachio P (1990) Characterization of four novel ras-like genes expressed in a human teratocarcinoma cell line. Mol Cell Biol 10:1793-1798.

Hoshijima M, Sah VP, Wang Y, Chien KR, Brown JH (1998) The low molecular weight GTPase Rho regulates myofibril formation and organization in neonatal rat ventricular myocytes. Involvement of Rho kinase. J Biol Chem 273:7725-7730.

Jin Z, Strittmatter SM (1997) Rac1 mediates collapsin-1-induced growth cone collapse. J Neurosci 17:6256-6263.

Kiryu S, Morita N, Ohno K, Maeno H, Kiyama H (1995a) Regulation of mRNA expression involved in Ras and PKA signal pathways during rat hypoglossal nerve regeneration. Mol Brain Res 29:147-156.

Kiryu S, Yao GL, Morita N, Kato H, Kiyama H (1995b) Nerve injury enhances rat neuronal glutamate transporter expression: identification by differential display PCR. J Neurosci 15:7872-7878. 
Kitahara T, Kiryu S, Ohno K, Morita N, Kubo T, Kiyama H (1994) Up-regulation of ERK (MAP kinase) and MEK (MAP kinase kinase) transcription after rat facial nerve transection. Neurosci Res 20:275-280.

Kozma R, Sarner S, Ahmed S, Lim L (1997) Rho family GTPases and neuronal growth cone remodelling: relationship between increased complexity induced by $\mathrm{Cdc} 42 \mathrm{Hs}$, Rac1, and acetylcholine and collapse induced by RhoA and lysophosphatidic acid. Mol Cell Biol 17:1201-1211.

Kranenburg O, Poland M, Gebbink M, Oomen L, Moolenaar WH (1997) Dissociation of LPA-induced cytoskeletal contraction from stress fiber formation by differential localization of Rho A. J Cell Sci 110:24172427.

Kuhn TB, Brown MD, Wilcox CL, Raper JA, Bamburg JR (1999) Myelin and collapsin-1 induce motor neuron growth cone collapse through different pathways: inhibition of collapse by opposing mutants of rac1. J Neurosci 19:1965-1975.

Leeuwen FN, Kain HE, Kammen RA, Michiels F, Kranenburg OW, Collard JG (1997) The guanine nucleotide exchange factor Tiam1 affects neuronal morphology; opposing roles for the small GTPases Rac and Rho. J Cell Biol 139:797-807.

Lin CH, Thompson CA, Forscher P (1994) Cytoskeletal reorganization underlying growth cone motility. Curr Opin Neurobiol [Erratum (1995)5:112]4:640-647.

Martini R (1994) Expression and functional roles of neural cell surface molecules and extracellular matrix components during development and regeneration of peripheral nerves. J Neurocytol 23:1-28.

Martini R, Schachner M (1988) Immunoelectron microscopic localization of neural cell adhesion molecules (L1, N-CAM, and myelinassociated glycoprotein) in regenerating adult mouse sciatic nerve. J Cell Biol 106:1735-1746.

Morita N, Kiryu S, Kiyama H (1996) p53-independent cyclin G expression in a group of mature neurons and its enhanced expression during nerve regeneration. J Neurosci 16:5961-5966.

Namikawa K, Su Q, Kiryu-Seo S, Kiyama H (1998) Enhanced expression of 14-3-3 family members in injured motoneurons. Mol Brain Res 55:315-320.
Namikawa K, Honma M, Abe K, Takeda M, Mansur K, Obata T, Miwa A, Okado H, Kiyama H (2000) Akt/PKB prevents injury-induced motor neuron death and accelerates axonal regeneration. $J$ Neurosci 20:2875-2886.

Neudauer CL, Joberty G, Tatsis N, Macara IG (1998) Distinct cellular effects and interactions of the rho-family GTPase TC10. Curr Biol $8: 1151-1160$

Nobes CD, Hall A (1995) Rho, rac, and cdc42 GTPases regulate the assembly of multimolecular focal complexes associated with actin stress fibers, lamellipodia, and filopodia. Cell 81:53-62.

Ridley AJ, Hall A (1992) The small GTP-binding protein rho regulates the assembly of focal adhesions and actin stress fibers in response to growth factors. Cell 70:389-399.

Ridley AJ, Paterson HF, Johnston CL, Diekmann D, Hall A (1992) The small GTP-binding protein rac regulates growth factor-induced membrane ruffling. Cell 70:401-410.

Sah VP, Hoshijima M, Chien KR, Brown JH (1996) Rho is required for Galphaq and alpha1-adrenergic receptor signaling in cardiomyocytes. Dissociation of Ras and Rho pathways. J Biol Chem 271:31185-31190.

Takano H, Komuro I, Oka T, Shiojima I, Hiroi Y, Mizuno T, Yazaki Y (1998) The Rho family G proteins play a critical role in muscle differentiation. Mol Cell Biol 18:1580-1589.

Tanabe K, Kiryu-Seo S, Nakamura T, Mori N, Tsujino H, Ochi T, Kiyama H (1998) Alternative expression of Shc family members in nerve-injured motoneurons. Mol Brain Res 53:291-296.

Tanabe K, Nakagomi S, Kiryu-Seo S, Namikawa K, Imai Y, Ochi T, Tohyama M, Kiyama H (1999) Expressed-sequence-tag approach to identify differentially expressed genes following peripheral nerve axotomy. Mol Brain Res 64:34-40.

Threadgill R, Bobb K, Ghosh A (1997) Regulation of dendritic growth and remodeling by Rho, Rac, and Cdc42. Neuron 19:625-634.

Yao GL, Kato H, Khalil M, Kiryu S, Kiyama H (1997) Selective upregulation of cytokine receptor subchain and their intracellular signalling molecules after peripheral nerve injury. Eur J Neurosci 9:10471054. 